\title{
Essais insecticides contre la Processionnaire du Pin (Thaumetopoea pityocampa SCHIFF) Action comparative de différentes spécialités commerciales Bactospéine, Dipel, Thuricide et Dimilin
}

\author{
G. DEMOLIN et A. MILLET \\ I.N.R.A., Station de Zoologie forestiere, \\ F 84000 Avignon
}

\begin{abstract}
Résumé
Les essais insecticides réalisés en jeune reboisement contre la Processionnaire du Pin, Thaumetopoea pityocampa SCHIFF à l'aide de trois produits à base de Bacillus thuringiensis: Bactospéine. Dipel, Thuricide et d'une spécialité à base de diflubenzuron : Dimilin, ont permis de préciser que les quatre formulations étudiées n'ont manifesté que peu de différence en ce qui concerne leur efficacité à moyen terme.
\end{abstract}

\section{1. - Introduction}

L'accroissement progressif des surfaces boisées en résineux a provoqué, depuis quelques années, une augmentation des dommages causés par la Processionnaire du pin qui, rappelons-le, sévit actuellement dans tous les pays du Bassin méditerranéen, à l'exception de l'Egypte et de la Libye.

Bien que plusieurs voies de recherche s'orientent actuellement vers la définition d'une nouvelle stratégie de lutte préventive par la mise en ouvre d'une sylviculture «adaptée»(GRISON, 1973; PARDE, 1974), il faut reconnaître que les responsables forestiers seront encore, pour plusieurs années, devant la nécessité d'utiliser des insecticides de façon plus ou moins drastique.

L'augmentation du nombre des spécialités efficaces proposées par les firmes commerciales peut les y aider mais il ne fait aucun doute qu'ils devront sans cesse rechercher une amélioration dans les méthodes d'essais et d'interventions ou, plus exactement, dans le choix des stratégies à mettre en œuvre. Ce choix dépendra de toute évidence des qualités propres à chaque insecticide, du type de forêt à protéger, mais surtout des connaissances acquises sur les mécanismes qui régissent les dynamiques des populations de la Processionnaire du pin.

En réalisant ces essais comparatifs, avec des produits aux performances assez semblables, notre propos est, en partie, de les y aider. 


\section{2. - Méthodes et techniques}

\subsection{Méthodes d'étude de la mortalité}

Compte tenu des particularités éthologiques de la Processionnaire du pin et principalement de son caractère social (DEMolin, 1967, 1969, 1971) deux méthodes d'estimation de la mortalité provoquée par des agents auxiliaires, ou un insecticide, peuvent être mises en cuvre.

\subsection{Table de mortalité}

Sur plusieurs lots d'arbres infestés par la Processionnaire du pin et groupés en échantillons représentatifs, on enregistre la mortalité des œufs et des chenilles à partir de prélèvements successifs des pontes et des colonies. La quantification est obtenue par différence entre le nombre d'oufs effectivement pondus sur chaque lot échantillon et le nombre d'individus retrouvés sur les mêmes lots. La succession des prélèvements (qui peuvent être très nombreux) permet d'établir des tables ou des courbes de mortalité. Au point de vue pratique la mise en application de cette méthode nécessite un choix très objectif de la zone d'étude. Il faut que le feuillage des arbres soit accessible dans tout son volume afin de retrouver facilement l'ensemble des pontes et ensuite l'ensemble des colonies. Ce qui limite l'expérimentateur aux jeunes forêts. Il faut aussi préparer le protocole d'étude très longtemps à l'avance afin d'une part de prendre connaissance de la population en place et d'autre part d'assurer le ramassage des pontes, de l'ensemble des lots prévus, immédiatement après la période d'éclosion.

\subsection{Dénombrement des nids d'hiver}

Le dénombrement des nids d'hiver, qui sont très visibles même sur les grands arbres, est une des bases d'observation qui a été la plus utilisée pour évaluer les niveaux de population au cours des études démographiques (Dusaussoy, Geri, 1969). Cette méthode qui prend toute sa valeur lorsqu'elle est utilisée en forêt âgée n'est pas, à notre avis, assez précise pour comparer l'efficacité à court terme des insecticides dont on sait, a priori, qu'ils ont des performances assez semblables.

\subsection{Zone d'intervention}

Ayant établi un choix prioritaire à la mise en application de la première méthode, la forêt qui a été retenue pour l'expérimentation est un reboisement de dix ans d'une superficie de 54 hectares. Il est situé au Nord-Est du département du Gard, dans la forêt communale de Lussan, à une altitude de 290 mètres.

La plantation est réalisée en majorité sur terrain nu à raison de 1350 plants/ hectare. Dans la partie nord la végétation naturelle a été conservée entre les rangs. Le boisement est dans son ensemble très facile d'accès.

L'essence dominante est le pin noir d'Autriche; essence qui, notons-le, sera la seule à être prise en considération dans nos relevés. Il existe cependant quelques cèdres en mélange dans tout le peuplement (4 p. 100), quelques rangées de sapins de 
Céphalonie dans la partie Sud et plusieurs rangs de pin de Saltzmann all Nord du boisement. La population de pins noirs avait, en 1975, une hauteur moyenne de 2,15 mètres $(\sigma=0,47)$.

\subsection{Choix des insecticides}

Le choix des insecticides utilisés s'est limité à quatre spécialités parmi celles dont l'emploi était déjà autorisé en France ou en cours d'homologation.

- La Bactospéine P.M. - poudre mouillable à base de Bacillus thuringiensis, sérotype I, Berliner, titrant 6000 U.A.A.K./mg, fabrication Rhône-Poulenc.

- Le Dipel W.P. - poudre mouillable à base de Bacillus thuringiensis, souche H.D. 1 du sérotype III' (3A-3B) Kurstaki titrant 8000 U.A.A.K./mg de fabrication Abbott et distribué en France par C.E.V.A.

- Le Thuricide H.P. Produit voisin du Dipel titre seulement 4000 U.A.A.K./ mg, de fabrication Sandoz.

- Le Dimilin - poudre mouillable à base de diflubenzuron (25 p. 100) 1-(4 chlorophenyl)-3-(2,6-difluorobenzoyl)-urée, insecticide de synthèse de fabrication Philips Duphar distribué en France par la Quinoléine.

\section{3. - La Processionnaire du pin dans la zone expérimentale}

Le boisement de Lussan est situé dans la zone optimale de l'aire de répartition de la Processionnaire du pin (Demolin, 1969; Huchon, Demolin, 1970) ce qui explique l'importance des dégats enregistrés depuis plusieurs années par les services forestiers du département du Gard.

Avant l'expérimentation, les populations n'avaient été observées que de façon occasionnelle et peu précise. Cependant en connaissance des données bioclimatologiques du département du Gard la Processionnaire ne pouvait suivre, sauf conditions climatiques exceptionnelles, qu'un cycle très classique : apparition des adultes courant juillet, durée d'incubation des oufs de 35 à 40 jours, évolution ralentie en hiver (au $4^{e}$ stade larvaire) et période des processions de nymphose en mars-avril.

\subsection{Relevés biologiques pré-expérimentaux}

3.11. Détermination de la période d'émergence des adultes, pré-estimation du niveau d'infestation et de la date d'intervention

La période de vol des adultes a été obtenue par piégeage lumineux (Piège Demolin). La campagne de capture a duré du 7 au 22 juillet, elle a été complétée par des «sondages» bi-hebdomadaires jusqua au 15 août. 
Les résultats sont représentés sur la figure 1 ; c'est une courbe caractéristique pour l'espèce en zone optimale de son aire de répartition. Les quelques captures du mois d'août correspondent à une vague tardive qui ne donne que rarement une descendance viable (prolongation de la durée d'incubation et difficulté de survie hivernale).

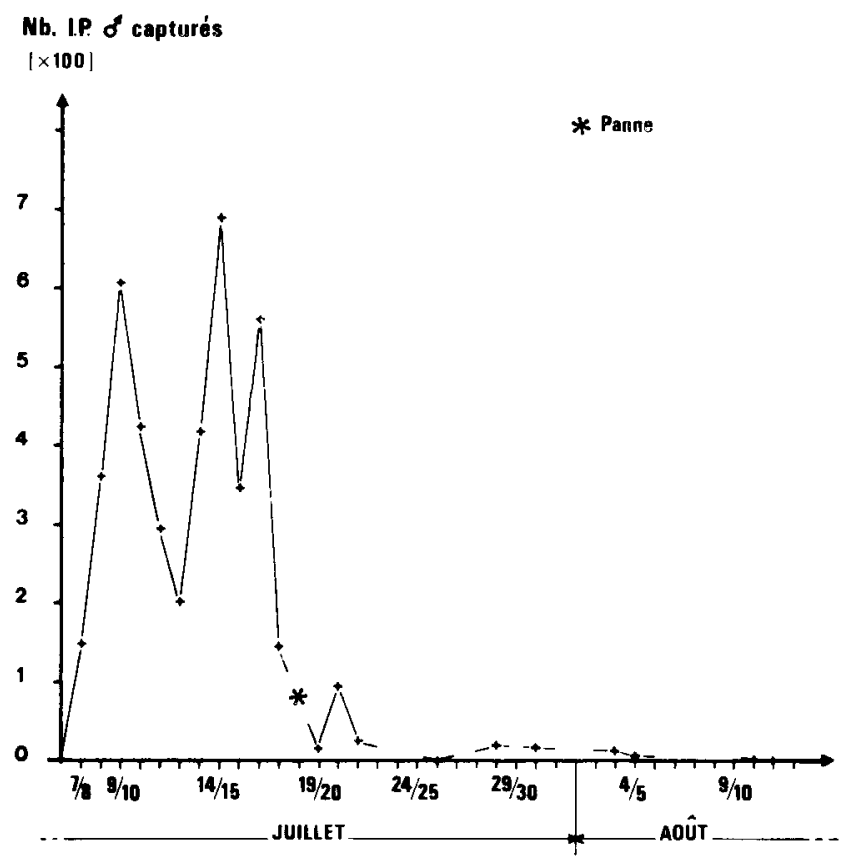

FIG. 1

Capture au piège lumineux

Capture in the light-trap

Nous retirons les éléments suivants :

- Le nombre des adultes capturés est relativement élevé, la population est donc suffisante pour exécuter les essais envisagés.

- La connaissance de la date moyenne des sorties d'adultes (15 juillet) et celle de la forme de la distribution indiquent que l'expérience pourra avoir lieu au cours de la deuxième semaine de septembre en prenant une durée moyenne d'incubation de 35 jours.

\subsection{Détermination du niveau d'infestation par dénombrement des pontes}

Pour compléter et préciser l'évaluation faite au piège lumineux nous avons dénombré les pontes présentes sur 280 arbres répartis sur l'ensemble du boisement; 991 pontes ont ainsi été récupérées soit une moyenne de 3,54 pontes par arbre. 
La partie nord et la partie centrale sont plus infestées que la partie sud de la forêt : respectivement $3,47,4,22$ et 2,77 pontes par pin.

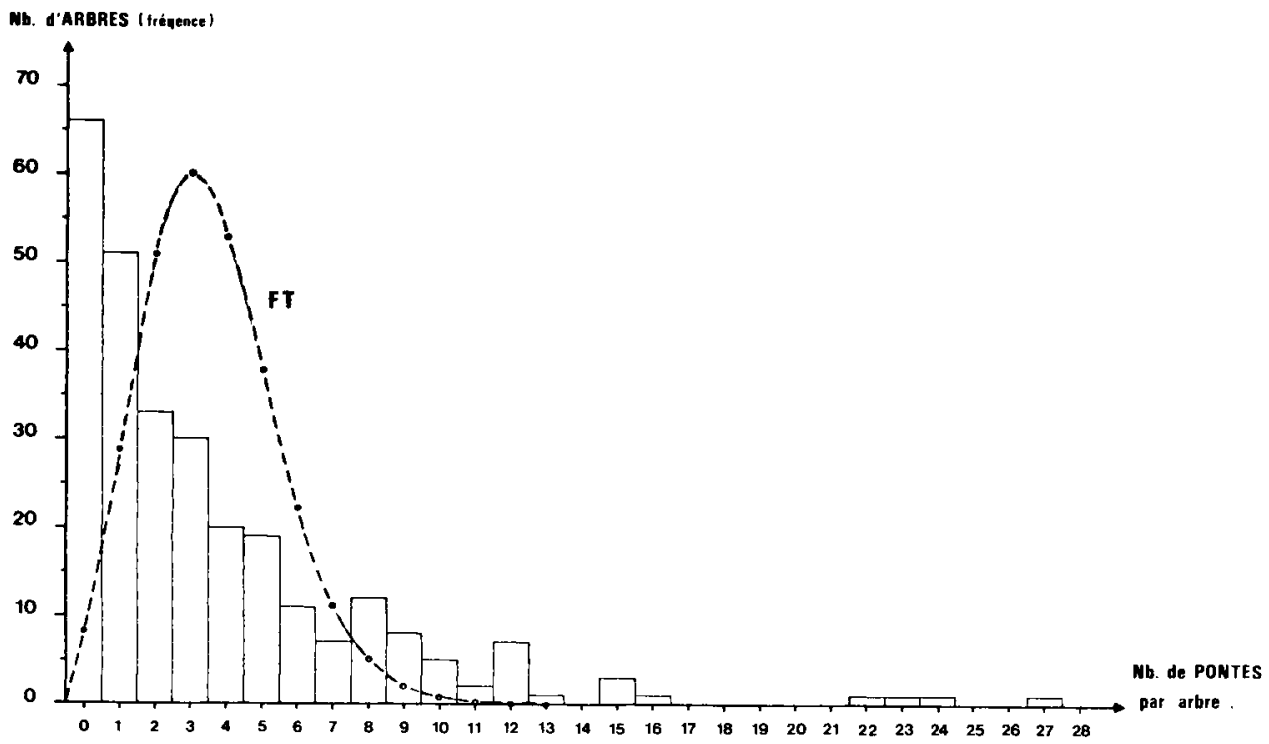

FIG. 2

Distribution du nombre de pontes par arbre pour un échantillon de $N=280$ pins (F.R.) histogramme et distribution de Poisson ajustée (F.T.)

Distribution of the number of egg-layings per tree for one representative sample of $N=280$ Pines.

Histogram (F.R.) and adjusted distribution (F.T.)

Si nous analysons de façon plus détaillée les résultats obtenus, voir figure 2 , nous constatons que tous les pins ne sont pas infestés de façon régulière ni au hasard. Ce résultat obtenu par comparaison de la fréquence observée et de la distribution théorique ajustée est la conséquence directe du comportement des femelles en fonction de la silhouette des arbres (Demolin, 1969).

\subsection{Détermination de la date précise d'intervention, éclosion des chenilles}

Toutes les pontes précédemment prélevées ont été conditionnées individuellement en tube de verre. Les lots ainsi constitués ont été placés sous abri extérieur au laboratoire du Mont Ventoux.

L'observation journalière des pontes nous a donné la courbe de fréquence des éclosions F.R. (premières chenilles écloses pour chaque ponte). La distribution a pour moyenne $\bar{x}=13,40$ (20 août) et pour écart type $\sigma=4,67$. Notons que nous avons groupé les données obtenues par classes de 3 jours pour le calcul de la fréquence théorique (F.T.) dans la figure 3. 


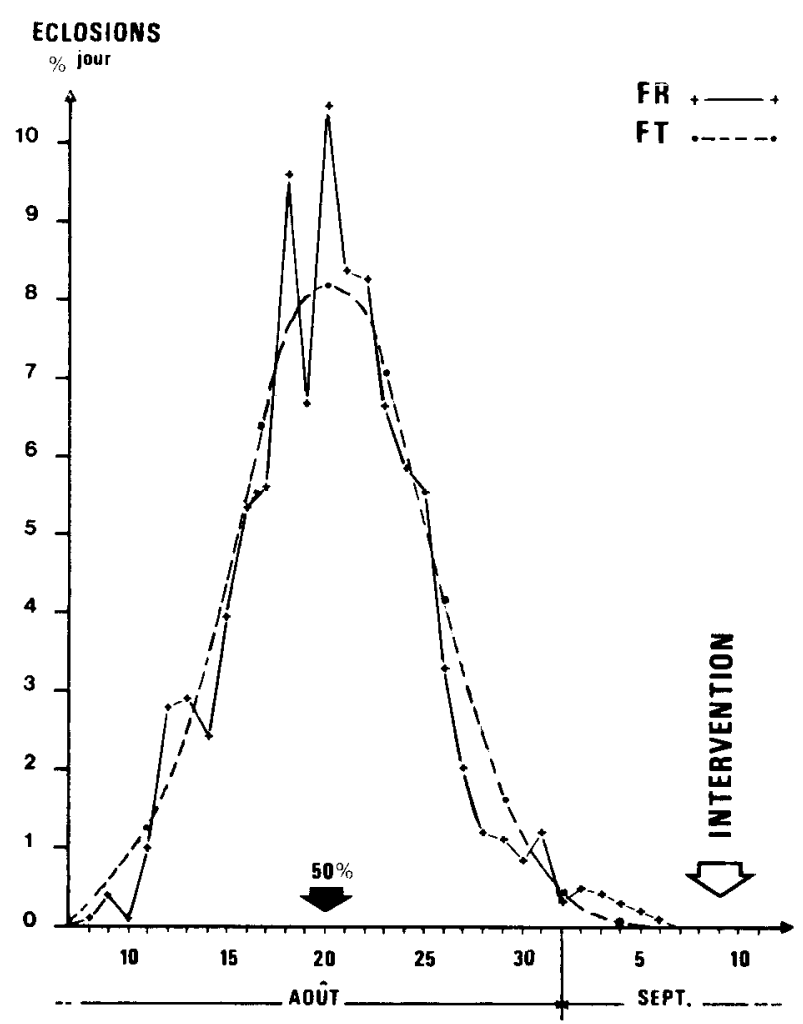

FIG. 3

Courbe d'éclosion des pontes au laboratoire (F.R.) et distribution ajustée (F.T.) Curve of the hatching out of the egg-layings in laboratory condition and adjusted distribution

Ces résultats montrent que :

- La durée moyenne d'incubation a été de 36 à 37 jours (écart entre les moyennes de la courbe de vol des adultes et celle de la courbe des éclosions). Cette durée a été d'ailleurs confirmée «in situ» en plaçant régulièrement des pontes de date connue sur le terrain.

- Les pontes ayant été récoltées entre le 7 et le 11 août (3.12.), donc bien après la période de vol du mois de juillet, on constate sur la courbe que le vol tardif n'a pas donné de descendance.

- La date du traitement pouvait être fixée pour le 9 septembre et cela dès le 15 août. Ce qui donnait une marge de temps suffisante pour assurer la préparation matérielle des opérations.

\subsection{Schéma d'évolution. Détermination des dates de prélèvement}

Les informations recueillies jusqu'au 15 août précisaient que la population était, comme prévu, bien «calée» par rapport au climat du biotope. Les larves suivraient donc un schéma d'évolution classique (DEMOLIN, 1969) que nous avons représenté sur la figure 4. 

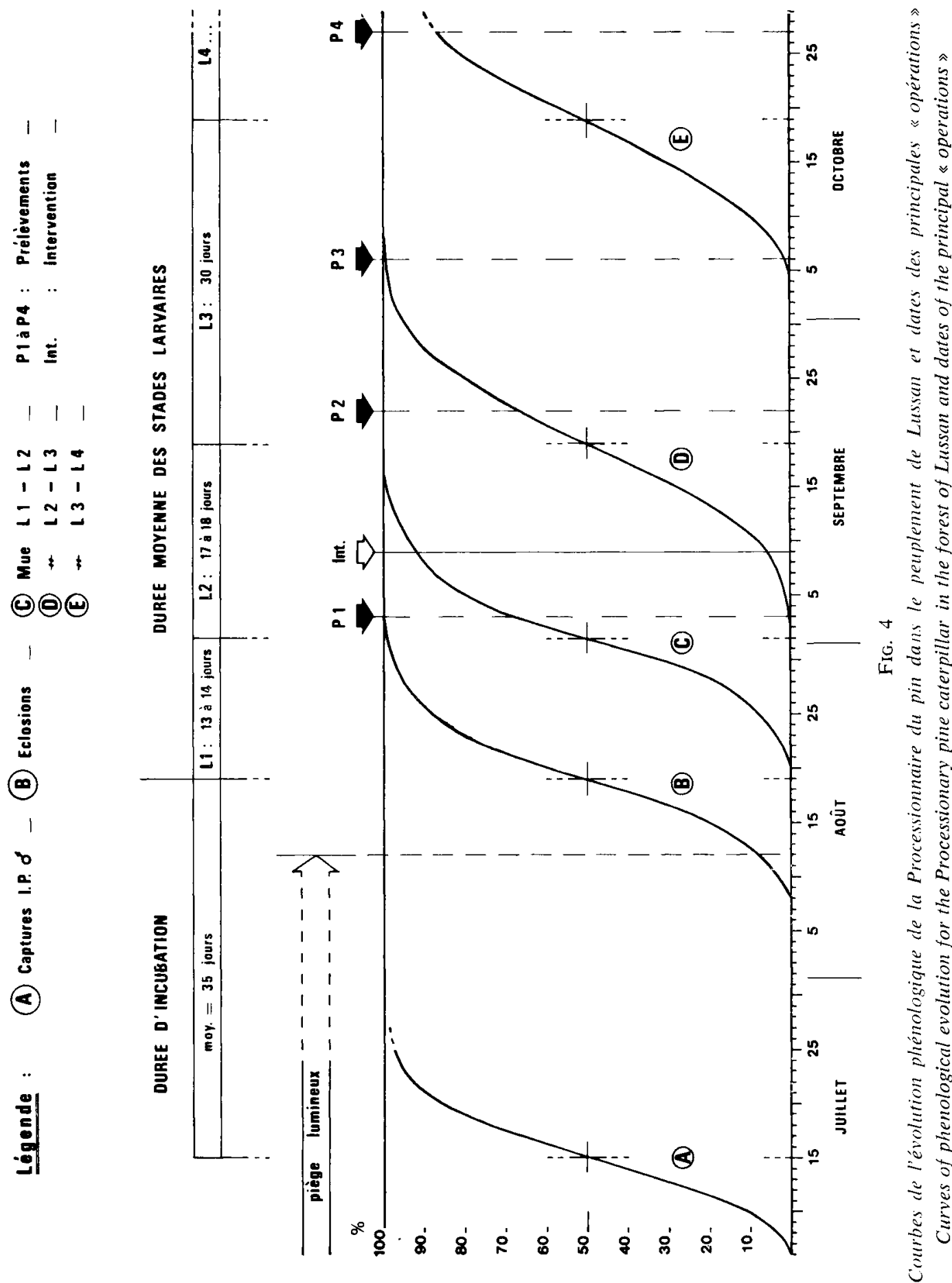
A partir de ce schéma théorique, établi le 15 août, nous pouvions mettre en place le calendrier opérationnel de traitement et de contrôle.

- L'intervention (jour J) a été prévue le 9 septembre lorsque la population serait aux $1^{\text {or }}$ et $2^{\circ}$ stades larvaires $(L 1, L 2)$ c'est-à-dire aux stades les plus favorables à l'efficacité des spécialités à base de Bacillus thuringiensis.

- Le premier prélèvement $(\mathrm{P} 1)$, ne concernant que les témoins, a été envisagé à J-6 soit le 3 septembre. Il avait pour but d'évaluer la mortalité naturelle au stade ouf et aux deux premiers stades larvaires pour la période située entre l'éclosion et l'intervention.

- Le deuxième prélèvement (P2) a été prévu lorsque la population serait au deuxième stade larvaire (L2) et en partie au troisième (L3) soit à $J+13$ le 22 septembre.

- Le troisième (P3) et le quatrième prélèvement (P4) ont été envisagés lorsque la population serait parvenue, respectivement au troisième et quatrième stade larvaire soit à $\mathrm{J}+28$, le 6 octobre, et à $\mathrm{J}+48$, le 27 octobre.

- Un cinquième prélèvement (P5) aurait lieu au printemps 1976 lorsque la population serait au cinquième stade larvaire (L5). La date ne pouvant pas être précisée à l'avance.

\subsection{Détermination de l'unité d'échantillonnage}

Compte tenu de linfestation existante et des connaissances progressivement acquises sur la population de la Processionnaire, nous avons admis qu'un lot de dix arbres tirés au hasard et supportant au moins une ponte, serait suffisant pour chaque prélèvement.

\section{4. - Opération proprement dite}

\subsection{Préparation de la zone expérimentale}

Notre intention étant de tester quatre produits, à deux doses différentes pour deux d'entre eux, le boisement a été découpé en onze parcelles. Sept d'entre elles ont été attribuées à chaque insecticide avec l'accord des responsables des différentes firmes commerciales : A1, A2, B1, B2, C1, C2, D (voir tableau 1) et les quatre autres ont été réservés comme témoins : T1, T2, T3, T4.

A la demande des expérimentateurs du Thuricide la parcelle A1 a été réservée à un essai au sol; de ce fait elle a été exclue de nos contrôles.

Afin d'éviter l'incidence des recouvrements accidentels, susceptibles de se produire lors du passage de l'hélicoptère, des placettes de 75 mètres de côté ont été aménagées au cour des parcelles pour y effectuer tous les contrôles expérimentaux. 
TABLEAU 1

Attribution des parcelles aux divers produits. Dose d'utilisation et témoins correspondants Atribution of allotments to the different products.

Employed quantity and corresponding control

\begin{tabular}{|c|c|c|c|c|}
\hline \multicolumn{2}{|c|}{ Parcelle traitée } & \multicolumn{2}{|c|}{ Produit } & \multirow{2}{*}{$\begin{array}{l}\text { Témoins } \\
\text { correspondants }\end{array}$} \\
\hline Dénomination & $\begin{array}{l}\text { Superficie } \\
\text { en ha }\end{array}$ & $\begin{array}{l}\text { Appellation } \\
\text { commerciale }\end{array}$ & $\begin{array}{c}\text { Dose } \\
\text { d'utilisation } \\
\text { à l'ha }\end{array}$ & \\
\hline A $1 \ldots \ldots \ldots$ & 1,20 & \multicolumn{2}{|c|}{ Thuricide (essai au sol) } & \\
\hline A 2 & 3,30 & Thuricide & $750 \mathrm{~g}$ & $\mathrm{~T} 1+\mathrm{T} 2$ \\
\hline B 1 & 1,80 & Thuricide & $1000 \mathrm{~g}$ & $\mathrm{~T} 2+\mathrm{T} 3$ \\
\hline B 2 & 7,50 & Dipel. & $1000 \mathrm{~g}$ & $\mathrm{~T} 2+\mathrm{T} 3$ \\
\hline C $1 \ldots$ & 1,80 & Dimilin & $600 \mathrm{~g}$ & $\mathrm{~T} 3+\mathrm{T} 4$ \\
\hline C 2 & 10,10 & Dimilin & $600 \mathrm{~g}$ & $\mathrm{~T} 3+\mathrm{T} 4$ \\
\hline $\mathrm{D} \ldots \ldots \ldots$ & 12,90 & Bactospéïnc & $1300 \mathrm{~g}$ & $\mathrm{~T} 3+\mathrm{T} 4$ \\
\hline
\end{tabular}

Les lots d'arbres échantillons ont été repérés dans chacune des placettes à raison de 50 pins dans les 4 placettes témoins ( 5 prélèvements $P 1$ à $P 5$ ) et 40 pins dans les 6 placettes traitées (4 prélèvements P2 à P5). Comme prévu les arbres ne portant pas de pontes ont été rejetés et remplacés par le pin le plus proche porteur d'au moins une ponte.

Enfin, au total, 440 arbres ont été marqués sur l'ensemble du boisement.

\subsection{Contrôles pré-traitement}

\subsection{Récolte de pontes}

A la fin de la période des éclosions, à partir đu 3 septembre, toutes les pontes présentes sur les 440 arbres échantillons ont été prélevées afin de déterminer avec précision la population initiale présente sur chaque pin.

Pour l'ensemble des placettes, 2046 pontes ont été récoltées, soit une moyenne de 4,65 pontes par arbre. Ce chiffre est supérieur à celui obtenu lors du premier prélèvement (voir 3.12.) qui était de 3,54. Cette différence s'explique par le rejet, lors du tirage, des pins ne possédant pas de ponte : si nous effectuons le même rejet pour le prélèvement initial soit 66 arbres sur 280 nous obtenons la moyenne de 4,63 , valeur pratiquement identique à celle qui vient d'être observée.

\subsection{Mortalité dans les témoins avant l'intervention}

Le 3 septembre, en même temps qu'il était procédé au ramassage des pontes dans toutes les placettes, les colonies présentes sur les pins de la première unité d'échantillonnage de T1, T2, T3 et T4 ont été prélevées et les chenilles dénombrées. 
La mortalité naturelle, rapportée au nombre d'oufs pondus, atteignait 49,96 p. 100 dont 36,26 p. 100 pour les aufs (parasites et prédatcurs) et 13,70 p. 100 pour les jeunes chenilles.

Si nous considérons l’ensemble des 2046 pontes prélevées et analysées une par une, la mortalité atteint 38,57 p. 100 pour les aufs, taux très voisin de celui déterminé ci-dessus pour un échantillon beaucoup plus restreint. Cette mortalité est due essentiellement, pour environ 30 p. 100, à l'action cumulée de deux parasites oophages : Tetrastichus servadei DOM. et Ooencyrtus pityocampae MERCET.

La fécondité s'avère également très faible avec une moyenne de 175,4 œufs par ponte: cette valeur est peut-être explicable par le fait que la population 19741975, qui était très importante, a pu souffrir d'une relative sous-alimentation.

\subsection{Composition de la population larvaire avant l'expérimentation}

Les dénombrements dans les placettes témoins nous donnent également la composition de la population par stades larvaires qui s'établit comme suit :

$1^{\mathrm{rr}}$ stade 38 p. 100

2 " stade 60 p. 100

3" stade 2 p. 100

c'est-à-dire que, sous ce rapport, se trouvaient réunies toutes les conditions biologiques optimales d'efficacité des insecticides choisis; particulièrement ceux à base de Bacillus thuringiensis.

\subsection{Intervention proprement dite}

\subsection{Préparation technique}

Parallèlement aux contrôles biologiques nécessaires aux essais la préparation technique de l'opération s'est poursuivie.

- Le choix de l'appareil a été arrêté à un hélicoptère BELL 47 G2 équipé d"une rampe de 50 gicleurs de $13 / 10^{\prime \prime}$ pour un débit moyen de 30 litres à l'hectare.

- Le découpage de la zone en placettes étant très complexe, un balisage précis, par cibles, a été mis en place de façon à permettre au pilote un recouvrement optimal (Plan directeur : figure 5).

- La qualité de l'épandage serait vérifiée par des cibles de canson noir posées sur k sol et des plaquettes de verre (protecteurs de diapositive); ces dernières ont été fixées sur chaque pin situé à laangle des placettes et à leur centre. Notons, qu'en chaque point, nous avons placé une plaquette verticale et une horizontale.

- les données météorologiques qui sont très importantes avant et après l'intervention ont été obtenues en collaboration avec le S.T.E.F.C.E. d'Avignon (I.N.R.A.).

\subsection{Conditions de l'intervention}

Commencé le 9 septembre, comme prévu, à 8 h 30 , l'épandage s'est poursuivi jusquà 13 heures sous de très bonnes conditions météorologiques : vitesse du vent de $4 \mathrm{~km} / \mathrm{h}$ et beau temps. 


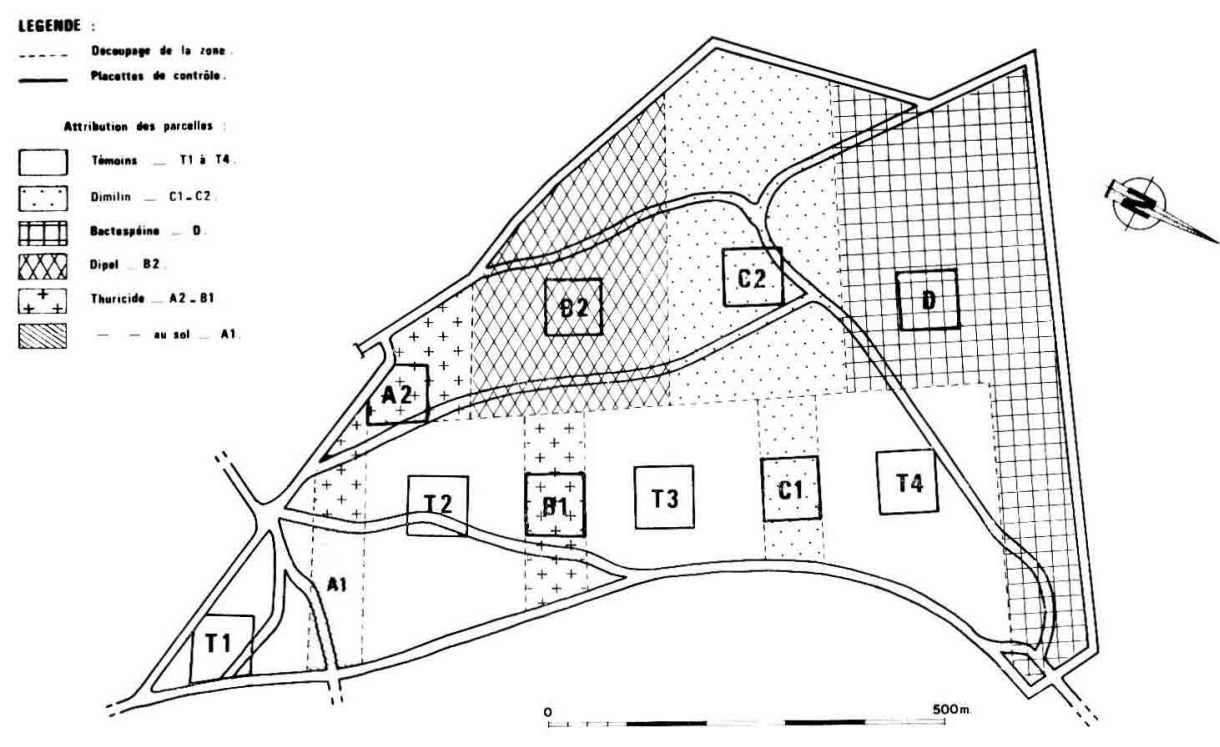

FIG. 5

Plan directeur. Découpage de la zone expérimentale et attribution des parcelles General plan. Cutting up of the experimental zone and attribution of allotments

Le contrôle à la fin de l'opération nous a confirmé la qualité et l'homogénéité de distribution de chacun des produits. Seul deux «manques» ont été relevés en bordure des placettes $\mathrm{Cl}$ (Dimilin) et $\mathrm{A} 2$ (Thuricide).

Un tirage photographique agrandi des dépôts de bouillie recueillis par les protecteurs de diapositives nous a montré une très bonne dispersion de chacune des spécialités excepté la Bactospéine (R.P.) dont le dépôt a conservé une structure plus grossière (gouttes de plus fort diamètre).

A signaler une pluie fine, $6,8 \mathrm{~mm}$ dans la nuit du 10 au 11 septembre après 23 heures et une pluie abondante, $50 \mathrm{~mm}$, dans la période du 11 au 15 . Les chenilles, encore très jeunes, donc de comportement alimentaire crépusculaire, ont pu ainsi effectuer deux prises alimentaires complètes avant tout début de lessivage des bouillies.

\section{5. - Résultats}

\subsection{Prélèvements}

Le protocole prévu le 16 août a été appliqué sans modification de date. Cependant le cinquième prélèvement, le 14 février 1976, a été perturbé en ce qui concerne les témoins par suite de l'importance de la population présente sur certains pins. 

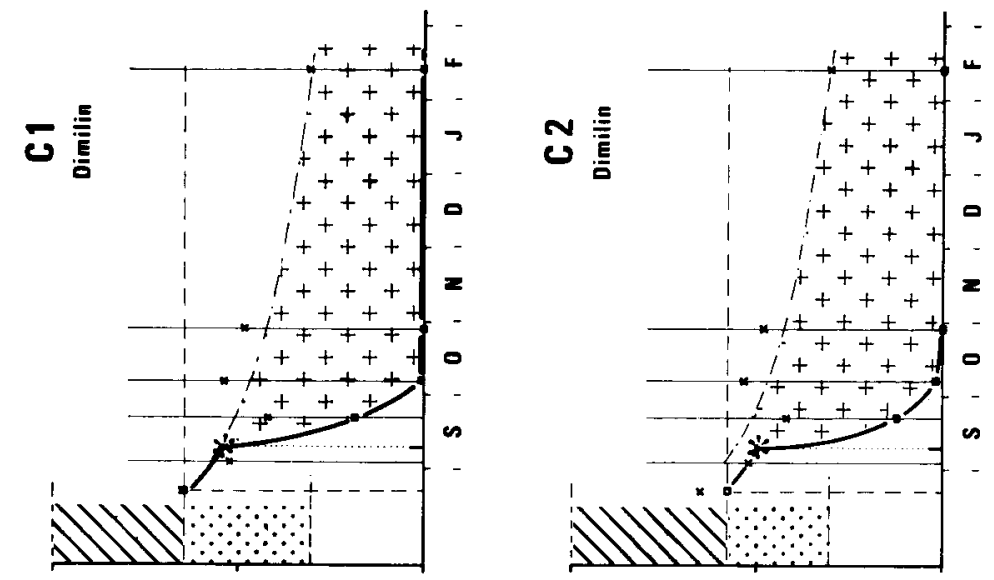

$\infty$ 흠
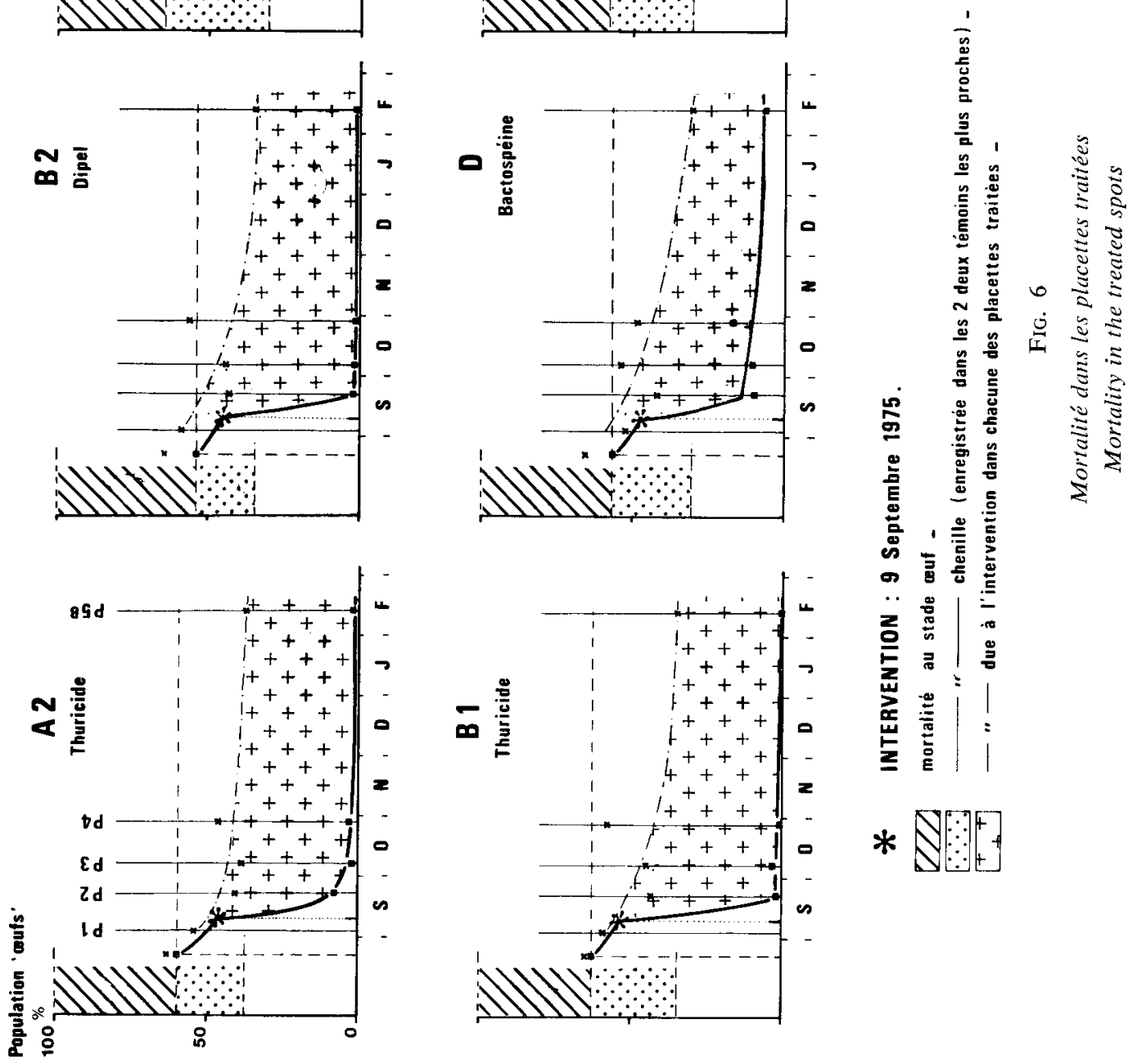
Pour l'analyse des résultats seuls les pins non entièrement défeuillés ont été pris en considération (prélèvement P5 B au lieu de P5).

Nous indiquons d'autre part, ci-après, la répartition par stades des populations de chenilles dans les placettes témoins, à chacune des dates de prélèvement.

\begin{tabular}{|c|c|c|c|c|c|c|c|}
\hline \multirow{2}{*}{ Prélèvements } & \multirow{2}{*}{ Dates } & \multirow{2}{*}{$\begin{array}{l}\text { Nombre } \\
\text { chenilles } \\
\text { prélevées }\end{array}$} & \multicolumn{5}{|c|}{ Stades larvaires en $\%$} \\
\hline & & & $\mathrm{L} 1$ & L2 & L3 & L4 & L5 \\
\hline $\mathrm{P} 1 \ldots \ldots \ldots \ldots$ & $03-09-75$ & 18120 & 38 & 60 & 2 & 0 & 0 \\
\hline $\mathrm{P} 2 \ldots \ldots$ & $22-09-75$ & 14732 & 0 & 45 & 55 & 0 & 0 \\
\hline P3 . & $06-10-75$ & 13267 & 0 & 1 & 99 & 0 & 0 \\
\hline P4 ..... & $25-10-75$ & 13158 & 0 & 0 & 17 & 83 & 0 \\
\hline P5 B & $14-02-76$ & 3968 & 0 & 0 & 0 & 8 & 92 \\
\hline
\end{tabular}

\subsection{Effets des produits} traitées.

La figure 6 donne les courbes d'évolution de la mortalité dans les placettes

Il apparaît de façon indéniable que tous les produits testés ont eu une très bonne efficacité contre la Processionnaire du pin. La Bactospéine (R.P.) montre une différence relativement sensible qui pourrait s'expliquer par une dispersion un peu moins fine, d'après les observations faites à la surface des protecteurs de diapositives. Il est vrai, qu'à la demande des représentants de la firme distributrice, la Bactospéine n'a pas été utilisée suivant les normes habituelles préconisées à cette époque ; c'est-àdire deux passages espacés, l'un de l'autre, de quinze jours.

Au contraire, on ne peut trouver de différences sensibles entre les trois autres spécialités, c'est-à-dire le Dipel, le Thuricide et le Dimilin (98 à 100 p. 100). En ce qui concerne ce dernier produit, agissant comme inhibiteur de mue par action sur la synthèse de la cuticule, son plein effet n'apparaît, comme c'était prévisible, qu'au $3^{\mathrm{e}}$ prélèvement.

Les 20 et 21 avril 1976, afin de compléter les résultats obtenus par les tables de mortalité, nous avons, sur 100 pins dans chaque placette, enregistré le nombre de nids par arbre et, par notation de 0 à 5 , le niveau de défoliation. Les résultats sont reportés sur la figure 7 . Ils confirment les conclusions tirées par l'autre méthode. Il est à noter que dans la parcelle traitée au Dimilin il nous a été impossible de retrouver un seul nid sur l'ensemble des arbres concernés.

D'un point de vue pratique, ces dernières observations nous indiquent qu'il suffit de quatre pontes pour qu'un pin noir soit défeuillé dans sa totalité lorsqu'il a $2,20 \mathrm{~m}$ de hauteur. 

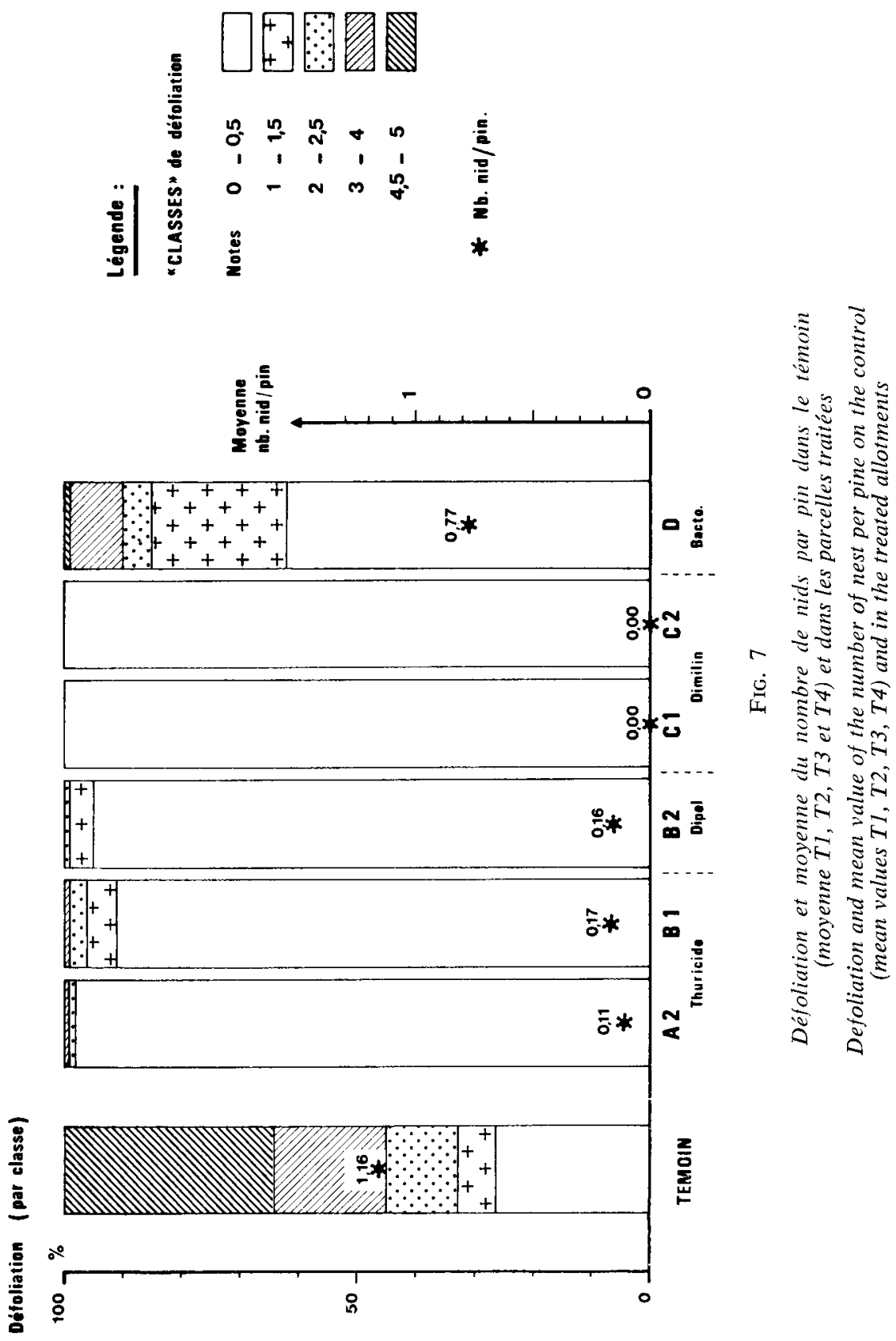


\title{
6. - Conclusion
}

Il est effectivement possible, se fondant sur un ensemble de relevés biologiques, d'établir les normes d'un protocole d'observations assurant une évaluation précise de l'efficacité d'un insecticide. Ceci n'étant applicable qu'en jeune peuplement.

Les résultats de cette expérience démontrent que, moyennant une détermination précise de la date diintervention, les produits à base de Bacillus thuringiensis montrent une excellente efficacité.

L'efficacité du Dimilin, excellente sur jeunes stades larvaires, n'apparaît dans sa totalité quavec retard. Ceci correspond bien au mode d'action du diflubenzuron, dont l'activité ne se manifeste entièrement qu'à l'occasion de la mue qui suit l'ingestion.

Les résultats quelque peu inférieurs de la Bactospéine s'expliquent peut-être par la nature de la charge. Depuis 1975, la firme COVAGRI, nouvelle détentrice de la marque, a modifié la formulation.

Nous signalons enfin que le reboisement à Lussan fut soumis en 1976 à une nouvelle intervention généralisée au Dimilin à effet de détruire le fort foyer de réinfestation laissé par les témoins de l'expérimentation de 1975. Des observations sur l'effet à terme de plusieurs années sont poursuivies depuis et feront l'objet d'une prochaine publication.

$$
\text { Reçu pour publication en aô̂t } 1980 .
$$

\section{Remerciements}

Ces expériences ont été réalisées en étroite collaboration avec l'Office National des Forêts, la Direction Départementale de l'Agriculture du département du Gard, et les spécialistes des différentes firmes de distribution des produits utilisés. Que tous les participants trouvent ici nos plus sincères remerciements.

\author{
Summary \\ Testing of insecticides against the Processionary caterpillar \\ (Thaumetopoea pityocampa Schiff) \\ Comparative action of different commercial specialities: \\ Bactospeine, Dipel, Thuricide and Dimilin
}

Insecticide trials carried out against processionary pine caterpillars from products containing Bacillus thuringiensis; Bactospeine, Dipel, Thuricide and diflubenzuron, Dimilin, applied with a method of comparative analysis by life table based on the knowledge of insect biology. There is very little difference between the four insecticides where the immediate efficacity is concerned in the short run. 


\section{Références bibliographiques}

Demolin G., 1967. Grégarisme et subsocialité chez Thaumetopoea pityocampa Schiff. Nid d'hiver - activité de tissage. Compte rendu du $V^{e}$ congrès de l'Union Internationale pour l'étude des insectes socianx, Toulouse, 5-10 juillet 1965, 69-77.

Demolin G., 1969. Comportement des adultes de Thaumetopoea pityocampa Schiff. Dispersion spatiale, importance écologique. Ann. Sci. for., 26 (1), 81-102.

Demolin G., 1969. Bioécologie de la Processionnaire du Pin, Thaumetopoea pityocampa Schiff. Incidence des facteurs climatiques. Colloque D.G.R.S.T., Pont-à-Mousson. Boll. Serv. Plagets for., XII, 23, 1-14.

Demolin G., 1971. Incidence de quelques facteurs agissant sur le comportement social des chenilles de Thaumetopoea pityocampa Schiff. (Lépidoptère) pendant la période des processions de nymphose. Répercussion sur l'efficacité des parasites. Colloque D.G.R.S.T., Pont-à-Mousson. Ann. Zool. Ecol. anim. (H.S.), «Lutte biologique en forêt », 33-56.

Dusausssoy G., Geri C., 1969. Etude des fluctuations du niveau de population de la Processionnaire du pin dans la vallée du Niolo en Corse (Thaumetopoea pityocampa Schiff.). Dispositif d'étude et premiers résultats. Ann. Sci. for., 26 (1), 103-125.

Grison P., 1970. Lucha microbiologia contra la «Procesionaria del pino». Bol. Serv. Plagas for., XIII, 26, 133-143.

Grison P., 1973. Lutte intégrée en forêt. Phytiatr. Phytopharm., 22, 229-248.

Huchon H., Demolin G., 1970. La bioécologie de la Processionnaire du pin. Dispersion potentielle - dispersion actuelle. Rev. for. fr., $\mathrm{n}^{\circ}$ spécial «Lutte biologique en forêt », 220-234.

PARDE J., 6-1974. Ravageurs, lutte intégrée et sylviculture. Rev. for. fr., XXVI, 419-426.

Ruperez A., 1968. Tecnicas y modalidades de aplicacion de preparationes microbiologicas. Bol. Serv. Plagas for., XI, 21, 47-58. 